\title{
Bidirectional associations between diet and body composition measures from 2 to 15 years: Longitudinal Study of Australian Children
}

\author{
Constantine E. Gasser ${ }^{1,2 *}$, Fiona K. Mensah ${ }^{2,3}$, Susan A. Clifford ${ }^{1,2}$, Jessica A. Kerr ${ }^{1,2}$, Raisa Cassim ${ }^{4,5}$ \\ and Melissa Wake ${ }^{1,2}$ \\ ${ }^{1}$ Centre for Community Child Health, Murdoch Children's Research Institute, Royal Children's Hospital, 50 Flemington Road, \\ Parkville, VIC 3052, Australia \\ ${ }^{2}$ Department of Paediatrics, Royal Children's Hospital, University of Melbourne, 50 Flemington Road, Parkville, \\ VIC 3052, Australia \\ ${ }^{3}$ Clinical Epidemiology and Biostatistics Unit, Murdoch Children's Research Institute, Royal Children's Hospital, 50 Flemington \\ Road, Parkville, VIC 3052, Australia \\ ${ }^{4}$ Allergy and Lung Health Unit, Centre for Epidemiology and Biostatistics, Melbourne School of Population and Global Health, \\ University of Melbourne, Parkville, VIC 3052, Australia \\ ${ }^{5}$ Gastro and Food Allergy Group, Murdoch Children's Research Institute, Royal Children's Hospital, 50 Flemington Road, \\ Parkville, VIC 3052, Australia
}

(Submitted 6 June 2018 - Final revision received 30 September 2018 - Accepted 7 October 2018)

\section{Abstract}

This study investigates how dietary patterns and scores are associated with subsequent BMI and waist:height ratio (WHtR), and how BMI and WHtR are associated with subsequent dietary patterns or scores, from 2-3 to 10-11 and 4-5 to 14-15 years of age. In the Longitudinal Study of Australian Children, height, weight and waist circumference were measured biennially in children, yielding BMI $z$-score and WHtR. Parents, latterly children, reported frequency of child consumption of $12-16 \mathrm{food} / \mathrm{drink}$ items during the previous $24 \mathrm{~h}$. At each wave, we empirically derived dietary patterns using factor analyses, and dietary scores based on the 2013 Australian Dietary Guidelines. We used structural-equation modelling to investigate cross-lagged associations ( $n$ 1972-2882) between diet and body composition measures in univariable and multivariable analyses. Dietary scores/patterns did not consistently predict WHtR and BMI $z$-score in the next wave, nor did BMI $z$-score and WHtR consistently predict diet in the next wave. The few associations seen were weak and often in the opposite direction to that hypothesised. The largest effect, associated with each standard deviation increase in BMI in wave 5 of the K cohort (age 12-13 years), was a 0.06 standard deviation estimated mean increase in dietary score (higher quality diet) in the subsequent wave $(95 \% \mathrm{CI} 0.02,0.11, P=0.003)$. Associations between dietary patterns/scores and body composition were not strongly evident in either direction. Better quantitative childhood dietary tools feasible for large-scale administration are needed to quantify how dietary patterns, energy intake and anthropometry co-develop.

Key words: BMI: Children: Dietary patterns: Dietary scores: Waist:height ratio

Obesity is a major public health issue. Approximately one-quarter of Australian children are overweight or obese and $7 \%$ are obese $^{(1)}$. A major driver of childhood obesity is when dietary energy intake exceeds energy expenditure. A major contributor to this excessive energy intake among children and adolescents is foods that are poor in dietary quality (e.g. higher in added sugar, saturated fat and/or salt) such as cakes, biscuits and processed meat products ${ }^{(2)}$. However, with childhood obesity interventions being largely ineffective in the population ${ }^{(3,4)}$, it is crucial that we better understand any bi-directionality of the relationship between children's body composition and dietary quality.
An alternative, and not mutually exclusive, hypothesis is that obesity drives subsequent diet ${ }^{(5)}$. Leptin, an adipocyte hormone, is involved in inhibiting dietary intake ${ }^{(5,6)}$. Leptin resistance often occurs as a result of obesity. This means that, compared with non-obese people, obese people cannot as easily respond to leptin signals or regulate dietary intake ${ }^{(5)}$, meaning that they may consume more foods.

It is possible that pathways from diet to body composition and from body composition to diet both operate simultaneously. Measures of body composition commonly include BMI and waist:height ratio (WHtR) (waist circumference $(\mathrm{cm})$

Abbreviations: LSAC, Longitudinal Study of Australian Children; WHtR, waist:height ratio.

* Corresponding author: C. E. Gasser, email constantine.gasser@mcri.edu.au 
divided by height $(\mathrm{cm})$ ), which is a proxy for total fat ${ }^{(7)}$. Bi-directionality between diet and body composition is important to consider for several reasons. In studies examining dietary determinants of body composition, reverse causality might occur, that is, body composition influencing diet ${ }^{(8,9)}$. Furthermore, it is important to determine the relative contribution of childhood diet to body composition, and how this contribution changes with child age through development, in order to inform the focus of future interventions.

Dietary patterns describe how foods group together in typical diets ${ }^{(10)}$. Dietary scores sum the frequency of foods consumed that are considered to have health benefits or detriments ${ }^{(10)}$. Previous longitudinal studies have mostly demonstrated null associations between dietary patterns or scores as determinants of BMI or WHtR during childhood and/or adolescence ${ }^{(11-14)}$. However, few studies have investigated both (i) whether diet is associated with subsequent measures of body composition (e.g. BMI or WHtR) and (ii) whether measures of body composition (e.g. BMI or WHtR) are associated with subsequent diet in children or adolescents. Within a population-based birth cohort of approximately 3500 Portuguese children ${ }^{(14)}$, an energy-dense foods pattern at 4 years of age was associated with higher BMI and WHtR at 7 years of age in girls but not in boys. They also found no evidence of adiposity measures at 4 years of age that were predictive of dietary patterns at 7 years of age ${ }^{(14)}$. In young children, the consumption of energy-dense foods at 2 years of age did not predict BMI $z$-score at 4 years of age, nor did BMI $z$-score at 2 years predict energy-dense food consumption at 4 years of age ${ }^{(15)}$.

Thus, there are significant gaps in what is known about bi-directional relationships between dietary quality and body composition across the full span of childhood. If strong relationships were found using low-burden, short self-reported dietary measures that are feasible for everyday use, then these could perhaps inform clinical or population guidance. This study aimed to investigate (i) how dietary patterns and scores are associated with subsequent BMI and WHtR, and (ii) how BMI and WHtR are associated with subsequent dietary patterns and scores, from ages $2-3$ to $10-11$ and $4-5$ to $14-15$ years, in the two population-representative cohorts of the Longitudinal Study of Australian Children (LSAC). Dietary scores were based on the 2013 Australian Dietary Guidelines recommendations for children, which encourage high numbers of daily servings of fruit, vegetables and milk products or alternatives; plenty of water; and a limited intake of fatty foods, sugary foods and sweetened drinks ${ }^{(16)}$. We hypothesised that (i) healthier dietary scores, higher adherence to a healthier diet (including frequent consumption of vegetables and fruit) and lower adherence to an unhealthier diet (including frequent consumption of sweetened drinks and savoury snacks) would predict lower BMI and WHtR in subsequent waves, and that (ii) lower BMI and WHtR would predict healthier dietary scores, higher adherence to a healthier diet and lower adherence to an unhealthier diet in subsequent waves.

\section{Methods}

\section{Recruitment and sampling}

This paper involved secondary analyses of observational data from LSAC. LSAC commenced in 2004; since then, it has collected data every 2 years from the B cohort (aged 0-1 years at wave 1 ) and the K cohort (aged $4-5$ years at wave 1$)^{(17,18)}$. This study used data from waves 1-6 of the B and K cohorts of LSAC, collected between 2004 and $2014^{(17)}$.

LSAC consisted of a general population-representative sample ${ }^{(19)}$. However, the most remote areas of Australia were excluded ${ }^{(19)}$. LSAC's sampling frame was the Medicare enrollment database ${ }^{(19)}$. Medicare is Australia's universal governmentfunded healthcare programme into which $98 \%$ of Australian children are enrolled by 12 months ${ }^{(19)}$. The sample of children included in LSAC was designed to represent Australia's states/ territories and consisted of an urban/rural mix ${ }^{(19)}$. LSAC used a two-stage clustered sampling design ${ }^{(19)}$. Briefly, this design involved the random selection of $311 / 3325$ (9.4\%) of Australian postcodes, after stratification by state/territory, capital city $v$. rest of state and large $v$. small population size ${ }^{(19)}$, followed by the random selection of an average of twenty children from each of these postcodes in the smaller states and territories (forty children in the larger states) ${ }^{(19)}$.

A total of 5107 and 4983 children were recruited into the B and $\mathrm{K}$ cohorts, respectively ${ }^{(19)}$, representing 57.2 and $50.4 \%$ uptake. Approximately 5000 children were recruited for each cohort so that the sample size was large enough so that even after several waves of data collection, detailed statistical analyses could be performed ${ }^{(19)}$. Online Supplementary Fig. S1 shows the retention and numbers across each of the LSAC waves. The Australian Institute of Family Studies Ethics Committee $^{(20)}$ approved each wave and families provided written informed consent to participate in the study.

\section{Procedures and measures}

In the LSAC, a variety of instruments, including questionnaires, have been used throughout the study ${ }^{(21)}$. The exact number of instruments differed by wave ${ }^{(21)}$. Relevant to these analyses, data collection methods consisted of audio computer-assisted interviews (for child ages 10 years or older); parent selfcomplete or leave behind questionnaires for both parents; face-to-face interviews, usually with 'Parent 1'; and physical measurements of the study child ${ }^{(21)}$. Parent 1 is the parent who knows the study child best, usually the biological mother ${ }^{(22)}$. These analyses used dietary and body composition data collected for children for waves 1-6 (ages 4-5 to 14-15 years) of the $\mathrm{K}$ cohort and waves $2-6$ (ages $2-3$ to $10-11$ years) of the $\mathrm{B}$ cohort.

Briefly, parents (for children aged 2-9 years) or children (aged 10 years or older) reported the number of times the study child consumed 12-16 individual or grouped food or drink items within the previous $24 \mathrm{~h}$ or yesterday (listed in online Supplementary Table S1) ${ }^{(23)}$. Our dietary tool was not previously validated. 


\section{Dietary scores}

We derived a dietary score for each child, at each wave, based mainly on recommended serves for a number of food groups, outlined in the 2013 Australian Dietary Guidelines ${ }^{(16)}$. First, frequency of consumption in the last $24 \mathrm{~h}$ was scored as 0,1 or 2 for each of seven food or drink categories: milk products or alternatives, vegetables, fruits and water (all positively coded), and fatty foods, sweetened drinks and sugary foods (all negatively coded). We summed the scores from each individual category to give an overall score, ranging from 0 to 14 , for each child at each wave, with higher scores reflecting a healthier diet. Readers are referred to our previous publication ${ }^{(23)}$ for further details on the derivation of the dietary scores.

\section{Dietary patterns}

We statistically derived dietary patterns for each wave using exploratory factor analyses ${ }^{(24)}$, using all $12-16$ food or drink items (shown in online Supplementary Table S1) to derive the factors or patterns. Readers are referred to our previous publication $^{(23)}$ for further details on the derivation of dietary patterns. We obtained 'healthy' and 'unhealthy' patterns or factors at each wave. The 'healthy' pattern was characterised by frequent consumption of vegetables and fruit in all waves, and water in most waves, each with high factor loadings of 0.3 or higher, reflecting these as foods or drinks that are most prominent in the 'healthy' factor ${ }^{(25)}$. The 'unhealthy' pattern was characterised by frequent consumption of sweetened drinks and savoury snacks in all waves, sausages or sausage rolls, hamburgers, hot dogs, meat pies, fruit juice and hot chips in most waves (each with high factor loadings of 0.3 or higher); and a high negative factor loading (below -0.3) for water consumption in six out of eleven waves. Finally, we used the means and standard deviations of each dietary variable to calculate continuous healthy and unhealthy dietary pattern scores for the healthy and unhealthy factors, respectively, for each child, with an approach known as regression scoring. Healthy dietary pattern scores ranged from -3.22 to 2.01 and unhealthy dietary pattern scores ranged from $-0 \cdot 96$ to $5 \cdot 27$.

\section{Child BMI z-score}

The child's weight was measured once using Salter Australia glass bathroom scales for wave 1, Salter Australia glass bathroom scales and HoMedics digital BMI bathroom scales for waves 2 and 3, and Tanita body fat scales for waves 4-6(22). Children were measured without shoes in light clothing. The child's height was measured using an Invicta stadiometer, from Modern Teaching Aids, for waves 1-3, and a laser stadiometer for waves $4-6^{(22)}$. Study researchers took two height measurements, and if the two measurements differed by at least $0.5 \mathrm{~cm}$, a third measurement ${ }^{(22)}$. The data file included the average of the two closest height measures ${ }^{(22)}$. For waves $2-6$ of the $\mathrm{B}$ cohort and 1-6 of the K cohort, child BMI was calculated as weight $(\mathrm{kg})$ divided by squared height $\left(\mathrm{m}^{2}\right)$. BMI was converted into BMI for age $z$-score (BMI $z$-score), based on Centers for Disease Control and Prevention growth charts ${ }^{(26)}$.

\section{Child waist:height ratio}

Waist circumference or girth was measured on the skin at the level of the child's navel, using a non-stretch dressmaker's tape ${ }^{(22)}$. Waist circumference was measured twice, or three times if the first two measurements differed by at least $0.5 \mathrm{~cm}^{(22)}$. The data file included the average of the two closest waist circumference measures ${ }^{(22)}$. For waves 2-6 of the $\mathrm{B}$ cohort and 1-6 of the K cohort, child WHtR was calculated as waist circumference $(\mathrm{cm})$ divided by height $(\mathrm{cm})$.

\section{Covariates}

Table 1 describes how covariates were measured in this study. Child covariates included indigenous background, language other than English spoken at home, television viewing, pubertal status, physical activity, age and sex, and whether the study child was a singleton or from a multiple birth. Parent covariates included parent $1 \mathrm{BMI}$, parent 1 age and parent 2 age. Other covariates were birth weight $z$-score (corrected for gestational age at birth), family/household socio-economic position and neighbourhood disadvantage.

\section{Statistical analysis}

We performed all statistical analyses using Stata/IC 14.2 (StataCorp LLC) and implementing survey methods, with the cross-sectional sampling weights from wave 1 of the $\mathrm{B}$ and $\mathrm{K}$ cohorts. In order to allow variables to be easily compared, we standardised dietary scores, 'healthy' patterns, 'unhealthy' patterns and WHtR to each have a mean of 0 and standard deviation of 1 . We investigated associations between each variable (all dietary measures, BMI $z$-score and WHtR) and the same variable in the subsequent wave, using linear regression analyses.

We performed cross-lagged analyses using the 'sem' command in Stata/IC 14.2. For these analyses, we included dietary scores, 'healthy' patterns or 'unhealthy' patterns as the exposure variable and BMI $z$-score or WHtR at the subsequent wave as the outcome variable, followed by BMI $z$-score or WHtR as the exposure variable and dietary scores/patterns at the subsequent wave as the outcome variable (a conceptual diagram of our analyses is provided as online Supplementary Fig. S2). We identified covariates from directed acyclic graphs and previous studies $^{(11-13,15,32-39)}$. The covariates included in cross-lagged analyses were child indigenous status, language other than English spoken at home, television viewing, pubertal status, physical activity, age and sex; birth weight $z$-score (corrected for gestational age at birth); and family/household socioeconomic position. We accounted for correlations between dietary and body composition variables from subsequent waves, using the 'cov' option. In order to ensure that crosslagged analyses that required a reduced sample (i.e. data from waves 2-6 of the B cohort or waves 1-6 of the K cohort) were consistent with wave-on-wave findings with more complete samples, we additionally conducted wave-on-wave multivariable linear and Tobit regression analyses between dietary and body composition measures. Drawing on prior 
Table 1. Longitudinal Study of Australian Children (LSAC) covariates and their handling for this study

\begin{tabular}{|c|c|}
\hline Covariate & Measure and additional information \\
\hline Socio-economic position & $\begin{array}{l}\text { LSAC provides a widely used composite socio-economic position variable at each wave that averages } \\
\text { information on parental occupational status, annual family income and parental educational attainment, } \\
\text { described previously }(27) \text {. We separated socio-economic position into quintiles, also using sampling } \\
\text { weights from wave } 1\end{array}$ \\
\hline Child indigenous background & $\begin{array}{l}\text { Parent } 1 \text { was asked whether the study child was of Aboriginal or Torres Strait Islander origin. We } \\
\text { dichotomised indigenous status into: 'yes' for children who were of Aboriginal and/or Torres Strait Islander } \\
\text { origin, and 'no' for children who were not }\end{array}$ \\
\hline $\begin{array}{l}\text { Child language other than English spoken } \\
\text { at home }\end{array}$ & $\begin{array}{l}\text { Parent } 1 \text { was asked whether the study child speaks a language other than English at home. Similar to } \\
\text { Renzaho et al. }{ }^{(28)}, \text { we grouped languages into: 'English only' and 'Other language' }\end{array}$ \\
\hline TV viewing & $\begin{array}{l}\text { The number of minutes on a typical (i) weekday and (ii) weekend day that the study child watches TV, DVDs } \\
\text { or videos at home was recorded. We calculated a continuous measure of the average daily TV, DVD or } \\
\text { video viewing hours, and classified this variable into two categories: ' }<2 \mathrm{~h} / \mathrm{d} \text { ' and ' } 2 \text { or more } \mathrm{h} / \mathrm{d} \text { ' }\end{array}$ \\
\hline Pubertal status & $\begin{array}{l}\text { For wave } 5 \text { of the B cohort and wave } 3 \text { of the } \mathrm{K} \text { cohort (i.e. child age } 8-9 \text { years), we dichotomised pubertal } \\
\text { development into 'no definite indication' and 'any definite indication', based on questions relating to: skin } \\
\text { changes (e.g. pimples), body hair growth, breast growth (females only) and adult type body odour } \\
\text { (K cohort only). For waves } 4 \text { and } 5 \text { of the } \mathrm{K} \text { cohort, we derived the categorical version of the Pubertal } \\
\text { Development Scale, which has been described previously }{ }^{(29)} \text {. This scale was based on questions relating } \\
\text { to body hair growth, voice deepening and growing hair on the face for males, and body hair growth, breast } \\
\text { development and menstruation for females. We classified response options into 'prepubertal', 'early } \\
\text { pubertal', 'midpubertal' and 'late pubertal/postpubertal' }\end{array}$ \\
\hline Child physical activity & $\begin{array}{l}\text { Parents were asked what the study child usually does when he/she has a choice about how to spend free } \\
\text { time. Response options were 'usually chooses inactive pastimes', 'usually chooses active pastimes' and } \\
\text { 'just as likely to choose active as inactive pastimes' }\end{array}$ \\
\hline Child age & In months. We separated child age into quartiles \\
\hline Child sex & Male/female \\
\hline $\begin{array}{l}\text { Birth weight } z \text {-score, corrected for } \\
\text { gestational age at birth }\end{array}$ & $\begin{array}{l}\text { Calculated using the 'zanthro' function in Stata/IC } 14.2 \text { (StataCorp LLC). We used the UK WHO Preterm } \\
\text { Growth Charts }^{(30)} \text { and adjusted for gestational age. We classified birth weight } z \text {-score into quartiles }\end{array}$ \\
\hline Parent $1 \mathrm{BMI}$ & $\begin{array}{l}\text { Parent BMI was calculated as weight }(\mathrm{kg}) \text { divided by squared height }\left(\mathrm{m}^{2}\right) \text {, based on self-reported weight and } \\
\text { height. Pregnant women were asked to record their usual weight when not pregnant. We classified parent } \\
1 \mathrm{BMI} \text { into four categories: 'underweight', 'normal weight', 'overweight' and 'obese' }\end{array}$ \\
\hline $\begin{array}{l}\text { Socio-economic Indexes for Areas } \\
\text { neighbourhood disadvantage }\end{array}$ & $\begin{array}{l}\text { The census-derived Australian Bureau of Statistics Index of Relative Socio-economic Disadvantage } \\
\text { described previously }{ }^{(31)} \text {. We classified Socio-economic Indexes for Areas neighbourhood disadvantage } \\
\text { as quintiles, also using sampling weights from wave } 1\end{array}$ \\
\hline Parent 1 , parent 2 age & $\begin{array}{l}\text { Parents reported their age at last birthday. We classified parental ages as: ' }<25 \text { ', ' } 25-29 \text { ', ' } 30-34 \text { ' and ' } 35+\text { ' } \\
\text { years for parent } 1 \text { in wave } 1 \text { of the B cohort; and ' }<30 \text { ', ' } 30-34 \text { ', ' } 35-39 \text { ' and ' } 40+\text { ' years for parent } 2 \text { in } \\
\text { wave } 1 \text { of the B cohort, and parents } 1 \text { and } 2 \text { in wave } 1 \text { of the K cohort }\end{array}$ \\
\hline Singleton/multiple birth & $\begin{array}{l}\text { Parent } 1 \text { was asked whether the study child was a single birth, a twin, a triplet or more. Response options } \\
\text { classified into 'single birth' and 'multiple birth' }\end{array}$ \\
\hline
\end{tabular}

TV, television; DVD, digital video disc.

literature ${ }^{(12,15,33,39)}$, we also conducted sensitivity analyses, which additionally included parent $1 \mathrm{BMI}$ as a covariate, and further sensitivity analyses, which additionally included parent 1 BMI, parent 1 age, parent 2 age, neighbourhood disadvantage and whether the study child was a singleton or from a multiple birth as covariates. The LSAC dataset is available to researchers (see www.growingupinaustralia.gov.au).

\section{Results}

\section{Descriptive characteristics}

Table 2 shows the baseline characteristics of the study sample, by cohort. There were approximately equal numbers of girls and boys, and children in both cohorts had mean BMI $z$-scores and WHtR of $0 \cdot 5$. The mean overall dietary scores were 10.3 and 9.7 out of a possible 14 points for children in the $\mathrm{B}$ and $\mathrm{K}$ cohorts, respectively, indicating that children in both cohorts generally had reasonably healthy diets at baseline. At age $8-9$ years, $86.3 \%$ of the B cohort and $88.9 \%$ of the $\mathrm{K}$ cohort children had not yet commenced puberty.
In both cohorts, mean BMI $z$-scores and WHtR did not change much across all of childhood and early adolescence (online Supplementary Table S2). The distribution of dietary variables was also similar at each wave of both cohorts (online Supplementary Table S2). A total of 1972-2882 children had complete data from waves 2-6 of the B cohort or all waves of the $\mathrm{K}$ cohort, so were included in crosslagged analyses. Wave-on-wave multivariable regression analyses included 2642-4100 children.

\section{Associations between dietary and body composition measures}

In linear regression analyses, for both cohorts we observed strong associations for each variable (all dietary measures, BMI $z$-score and WHtR) with the same variable in the subsequent wave (all $P<0 \cdot 001$; results not shown). Figs. 1 and 2 show the cross-lagged associations between dietary and body composition measures from multivariable analyses, accounting for correlations between dietary and body composition variables from subsequent waves. Numbers at the top of each panel are 
Table 2. Baseline characteristics* of the sample, by cohort (Mean values and standard deviations; percentages)

\begin{tabular}{|c|c|c|}
\hline Variable & $\begin{array}{c}\text { B cohort }(0-1 \\
\text { years) }(n 3996- \\
5107 \dagger)\end{array}$ & $\begin{array}{c}\text { K cohort (4-5 } \\
\text { years) ( } n 3847- \\
4983+)\end{array}$ \\
\hline \multicolumn{3}{|l|}{ Child age (years) } \\
\hline Mean & 0.7 & $4 \cdot 8$ \\
\hline SD & 0.2 & 0.2 \\
\hline Child sex (male, \%) & $51 \cdot 1$ & $51 \cdot 2$ \\
\hline Child has indigenous background (\%) & 4.9 & 3.9 \\
\hline $\begin{array}{l}\text { Child speaks only English at home } \\
(\%)\end{array}$ & $88 \cdot 9$ & $87 \cdot 6$ \\
\hline Child singleton birth (\%) & 96.9 & $97 \cdot 2$ \\
\hline Child television viewing $<2 \mathrm{~h} / \mathrm{d}(\%) \ddagger$ & $48 \cdot 9$ & $50 \cdot 1$ \\
\hline \multicolumn{3}{|l|}{ Child physical activity $(\%) \S$} \\
\hline Usually chooses inactive pastimes & $19 \cdot 9$ & $25 \cdot 9$ \\
\hline $\begin{array}{l}\text { Just as likely to choose active as } \\
\text { inactive pastimes }\end{array}$ & $44 \cdot 4$ & $44 \cdot 9$ \\
\hline Usually chooses active pastimes & $35 \cdot 7$ & $29 \cdot 2$ \\
\hline \multicolumn{3}{|l|}{ Child weight status (\%)§ } \\
\hline Underweight & $5 \cdot 3$ & $5 \cdot 2$ \\
\hline Normal & 71.6 & $74 \cdot 2$ \\
\hline Overweight & $18 \cdot 6$ & $15 \cdot 1$ \\
\hline Obese & 4.6 & 5.5 \\
\hline \multicolumn{3}{|l|}{ Child BMI z-score§ } \\
\hline Mean & 0.5 & 0.5 \\
\hline SD & $1 \cdot 1$ & $1 \cdot 0$ \\
\hline \multicolumn{3}{|l|}{ Child waist:height ratio§ } \\
\hline Mean & 0.5 & 0.5 \\
\hline SD & $0 \cdot 0$ & 0.0 \\
\hline \multicolumn{3}{|l|}{ Child overall dietary score $\S$} \\
\hline Mean & $10 \cdot 3$ & 9.7 \\
\hline SD & $2 \cdot 1$ & $2 \cdot 2$ \\
\hline \multicolumn{3}{|l|}{ Child healthy factor score§ } \\
\hline Mean & 0.0 & 0.0 \\
\hline SD & 0.6 & 0.7 \\
\hline \multicolumn{3}{|l|}{ Child unhealthy factor score§ } \\
\hline Mean & 0.0 & 0.0 \\
\hline SD & 0.7 & 0.7 \\
\hline \multicolumn{3}{|l|}{$\begin{array}{l}\text { Socio-economic Indexes for Areas } \\
\text { neighbourhood disadvantage }(\%)\end{array}$} \\
\hline Quintile 1 (least disadvantaged) & $19 \cdot 2$ & $18 \cdot 1$ \\
\hline Quintile 2 & $17 \cdot 0$ & $18 \cdot 0$ \\
\hline Quintile 3 & $20 \cdot 3$ & $19 \cdot 8$ \\
\hline Quintile 4 & $21 \cdot 0$ & $20 \cdot 7$ \\
\hline Quintile 5 (most disadvantaged) & $22 \cdot 6$ & 23.4 \\
\hline \multicolumn{3}{|l|}{ Parent 1 age (years) } \\
\hline Mean & $31 \cdot 0$ & $34 \cdot 6$ \\
\hline $\mathrm{SD}$ & $5 \cdot 7$ & 5.5 \\
\hline \multicolumn{3}{|l|}{ Parent 2 age (years) } \\
\hline Mean & $33 \cdot 8$ & $37 \cdot 4$ \\
\hline $\mathrm{SD}$ & $6 \cdot 1$ & $6 \cdot 2$ \\
\hline \multicolumn{3}{|l|}{ Parent 1 weight status (\%) } \\
\hline Underweight & $11 \cdot 7$ & $10 \cdot 5$ \\
\hline Normal & 43.9 & $45 \cdot 6$ \\
\hline Overweight & $25 \cdot 7$ & $26 \cdot 5$ \\
\hline Obese & $18 \cdot 6$ & $17 \cdot 4$ \\
\hline
\end{tabular}

* All estimates are weighted.

† Sample sizes differed for each variable.

¥ Measured at wave 2 (data not collected at wave 1).

$\S$ Measured at wave 2 of the $B$ cohort and wave 1 of the $K$ cohort.

coefficients representing the estimated mean increase or decrease in BMI $z$-score (Fig. 1) or standardised WHtR (Fig. 2) associated with a 1SD increase in each dietary measure. Numbers at the bottom of each panel are coefficients representing the estimated mean increase or decrease in each standardised dietary measure associated with a 1SD increase in BMI (Fig. 1) or WHtR (Fig. 2). Online Supplementary Tables
S3-S6 additionally provide 95\% CI and $P$-values for coefficients.

Generally, we observed no evident associations between dietary measures and BMI $z$-score or WHtR in univariable and multivariable cross-lagged analyses (Figs. 1 and 2; online Supplementary Tables S3-S6; univariable results available upon request). Findings were highly replicable in both cohorts. Dietary scores, continuous 'healthy' pattern scores and continuous 'unhealthy' pattern scores generally did not predict BMI $z$-score in the subsequent wave; likewise BMI $z$-score did not predict any measures of diet in the subsequent wave (Fig. 1; online Supplementary Tables S3 and S4). In multivariable analyses, adjusting for covariates, the few associations we observed were small and tended to be in the reverse direction to those hypothesised, that is, higher BMI $z$-score was associated with very slightly higher dietary scores (i.e. better adherence to the Australian Dietary Guidelines ${ }^{(16)}$ ) and lower adherence to an unhealthy diet. For example, a 1SD increase in BMI in wave 5 of the $\mathrm{K}$ cohort was associated with an estimated mean improvement in the standardised dietary score measure of 0.06 units (95\% CI $0.02,0.11, P=0.003$ ) in the subsequent wave (Fig. 1; online Supplementary Table S4). Similarly, a 1sD increase in BMI in wave 5 of the $\mathrm{K}$ cohort was associated with an estimated mean decrease (improvement) in the standardised 'unhealthy' pattern score of 0.06 units $(95 \% \mathrm{CI}-0.09,-0.02, P=0.002)$ in the subsequent wave (Fig. 1; online Supplementary Table S4). Associations between dietary measures and BMI $z$-score remained similar in sensitivity analyses (results not shown). These analyses additionally included parent 1 BMI as a covariate, and then (in further sensitivity analyses) additionally included parent 1 BMI, parent 1 age, parent 2 age, neighbourhood disadvantage and whether the study child was a singleton or from a multiple birth as covariates.

In multivariable cross-lagged analyses, the few associations we observed between diet and WHtR were again small and inconsistent, but this time tended to be in the same direction to those hypothesised, that is, higher WHtR was associated with lower adherence to a healthy diet and generally associated with higher adherence to an unhealthy diet (Fig. 2; online Supplementary Tables S5 and S6). For example, a 1sD increase in WHtR in wave 4 of the K cohort was associated with an estimated mean decrease (worsening) in the standardised 'healthy' factor score of 0.07 units $(95 \% \mathrm{CI}-0.12,-0.02 ; P=0.007)$ in the subsequent wave. In both sets of sensitivity analyses, associations between dietary measures and WHtR generally remained similar (results not shown). Results for BMI $z$-score and WHtR remained similar in wave-on-wave multivariable linear and Tobit regression analyses (results not shown).

\section{Discussion}

\section{Statement of principal findings}

In a unique longitudinal and cross-sequential design, this study investigated the bi-directionality between diet and body composition measures using short dietary tools suitable for population use, in two cohorts and at several waves. We generally found that associations were not evident either between 
(a)

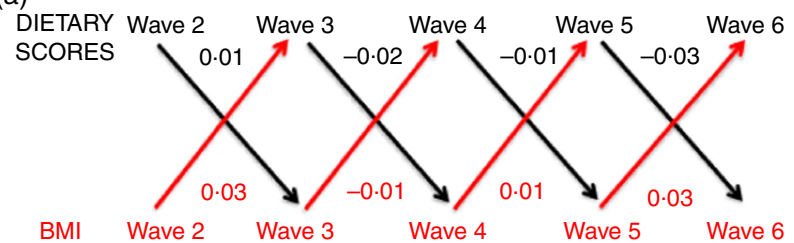

(c)

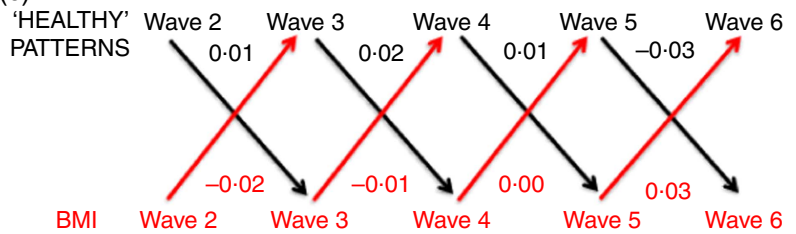

(e)

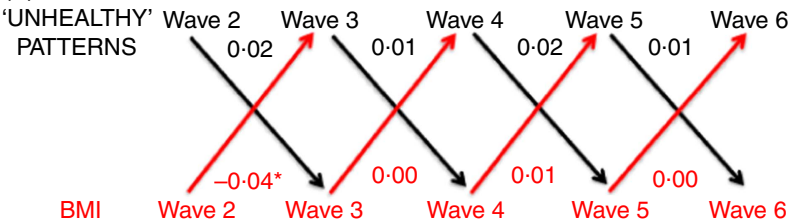

(b)

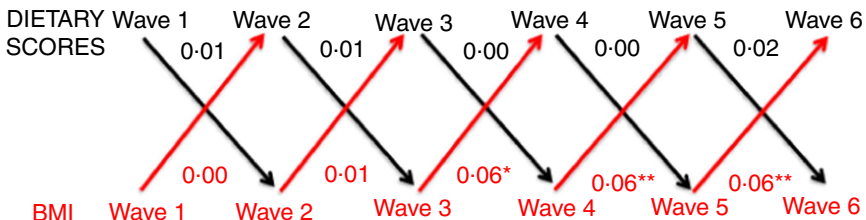

(d)

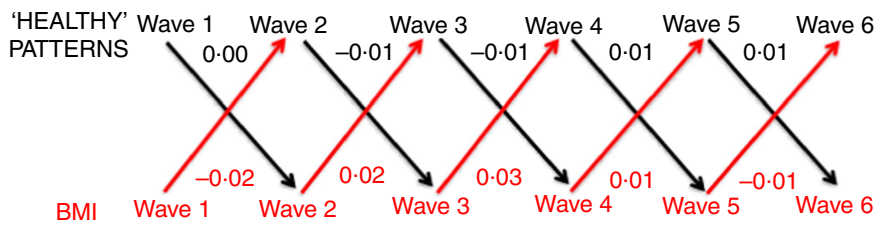

(f)

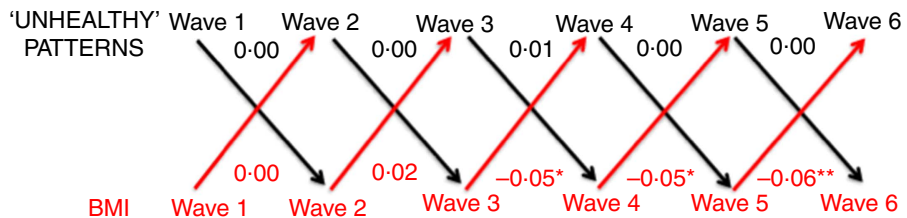

Fig. 1. Cross-lagged associations, derived from structural-equation modelling, between dietary scores and BMI for the $B$ cohort (a), dietary scores and BMI for the $K$ cohort (b), 'healthy' patterns and BMI for the B cohort (c), 'healthy' patterns and BMI for the K cohort (d), 'unhealthy' patterns and BMI for the B cohort (e), and 'unhealthy' patterns and BMI for the K cohort (f). Numbers denote coefficients, derived from structural-equation modelling. Dietary scores, 'healthy' patterns, 'unhealthy' patterns and BMI were each standardised to have a mean of 0 and standard deviation of $1 .{ }^{*} P<0.05,{ }^{* *} P<0.01$. Analyses adjusted for child indigenous status, language other than English spoken at home, television viewing, pubertal status, physical activity, age and sex; birth weight $z$-score and socio-economic position. We included covariates that remained relatively stable throughout the duration of the Longitudinal Study of Australian Children (child indigenous status, language other than English spoken at home and sex) from wave 1 of each cohort. For the remaining covariates which each had the potential to change over time, we included the measure from the same time point as the relevant exposure variable. If covariates were unavailable at a particular wave, we took them from the previous wave.

'healthy' patterns, 'unhealthy' patterns or dietary scores and BMI $z$-score or WHtR in cross-lagged analyses. The lack of association was consistent across both cohorts of children and between every set of biennial waves, and was robust to sensitivity analyses.

\section{Strengths and limitations}

A strength of this study is that it included consistent measures that were repeatedly collected over multiple waves spanning 10 years, allowing us to observe whether associations change with child age. Child height, weight and waist circumference in children were measured, rather than self-reported. In addition to the commonly-used BMI, the use of WHtR allowed us to determine whether our study findings were similar when considering an alternative measure of body composition. Replication of our findings across two cohorts of children increases confidence and reliability in the results ${ }^{(40)}$, and allows generalisation across a wider span of childhood than would otherwise have been possible. We use data from LSAC's national population-representative sample ${ }^{(19)}$, and employed survey methodology in our analyses to account for the multistage and clustered sampling design, and non-response ${ }^{(22)}$. Finally, rather than an alternative approach of studying individual foods or drinks, we considered two short, low-burden and holistic measures of diet that are well suited to population use and guidelines, specifically dietary scores and patterns. Studying dietary patterns allows us to determine which foods or drinks are eaten together in the diet and is not dependent upon the disease or restricted to recommendations given in dietary guidelines ${ }^{(10)}$. Dietary scores are developed based on previous research and may be easier for the public to understand ${ }^{(10)}$.

Despite the advantages of this short dietary measure, it also has limitations. Its brevity precludes capture of all dietary elements or all items included in the Australian Dietary Guidelines ${ }^{(16)}$. In assessing frequency of dietary intake and patterns in the previous $24 \mathrm{~h}$, it may not represent a child's habitual diet and does not capture energetic intake. Moreover, while children's diets may differ on a daily basis, the LSAC dataset does not indicate whether the previous $24 \mathrm{~h}$ recalled was a weekday or a weekend day. In addition, self- or parent-reported dietary intake can be limited by misremembering ${ }^{(41)}$ and underreporting ${ }^{(42)}$, with social desirability bias potentially masking true associations between unhealthy diets and body composition measures. Against this, our trajectories of these same dietary scores and patterns have previously demonstrated extremely large associations with both socieoconomic status ${ }^{(32)}$ and parental fruit and vegetable consumption ${ }^{(43)}$ in these same cohorts. While some participants were missing, results were very similar when we applied survey weights and conducted wave-on-wave regression analyses between dietary and body composition measures. A further limitation is that, although we also considered bi-directional associations between diet and WHtR, we acknowledge that WHtR may be a poorer indicator of central $\mathrm{fat}^{(7)}$ than previously thought ${ }^{(44)}$. Future studies should consider measures more specific to visceral adiposity. 
(a)

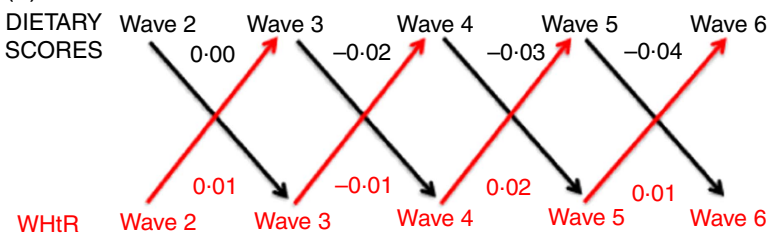

(c)

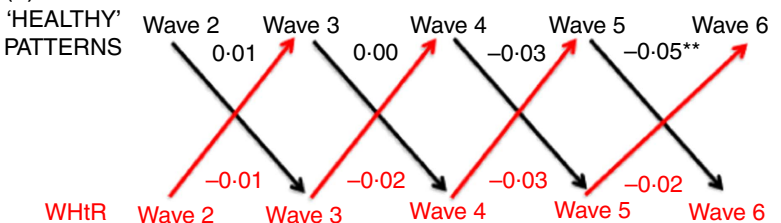

(e)

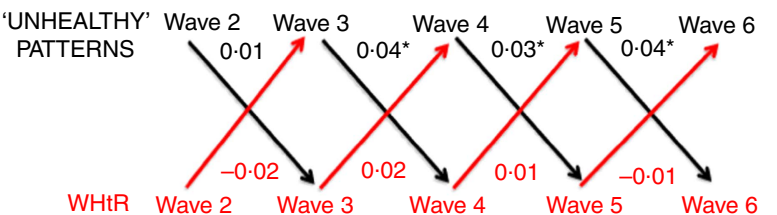

(b)

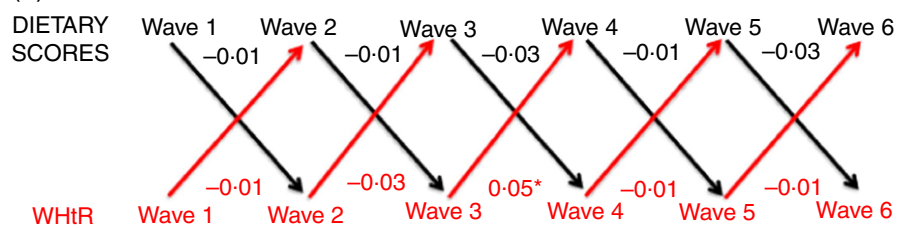

(d)

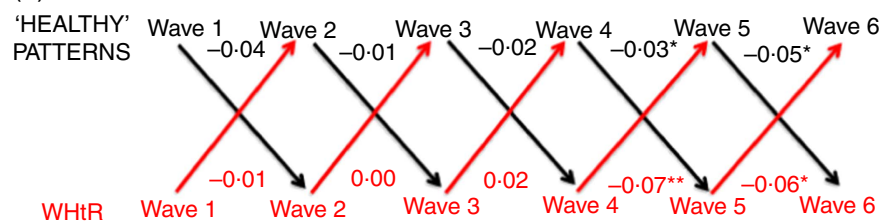

(f)

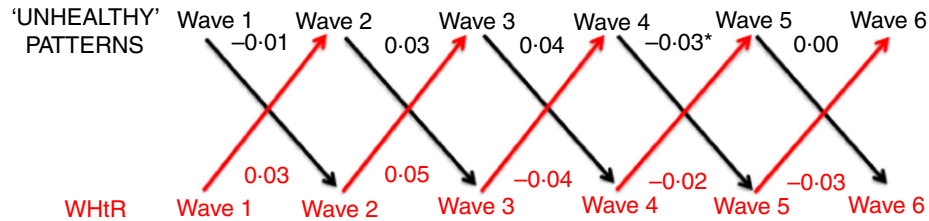

Fig. 2. Cross-lagged associations, derived from structural-equation modelling, between dietary scores and waist:height ratio (WHtR) for the $B$ cohort (a), dietary scores and WHtR for the K cohort (b), 'healthy' patterns and WHtR for the B cohort (c), 'healthy' patterns and WHtR for the K cohort (d), 'unhealthy' patterns and WHtR for the B cohort (e), and 'unhealthy' patterns and WHtR for the K cohort (f). Numbers denote coefficients, derived from structural-equation modelling. Dietary scores, 'healthy' patterns, 'unhealthy' patterns and WHtR were each standardised to have a mean of 0 and standard deviation of $1 .{ }^{*} P<0.05,{ }^{* *} P<0.01$. Analyses adjusted for child indigenous status, language other than English spoken at home, television viewing, pubertal status, physical activity, age and sex; birth weight $z$-score and socio-economic position. We included covariates that remained relatively stable throughout the duration of the Longitudinal Study of Australian Children (child indigenous status, language other than English spoken at home and sex) from wave 1 of each cohort. For the remaining covariates which each had the potential to change over time, we included the measure from the same time point as the relevant exposure variable. If covariates were unavailable at a particular wave, we took them from the previous wave.

\section{Comparison with prior literature}

Our main finding of null associations between dietary scores/ patterns and measures of body composition aligns with the previous English and Australian studies in which dietary patterns or quality in early childhood or adolescence were not associated with $\mathrm{BMI}^{(11-13)}$. However, our study goes further in replicating across cohorts, spanning multiple ages and considering the bi-directionality of associations between diet and body composition. Previous studies that have considered the bi-directionality of associations between diet and body composition across shorter periods than our study have likewise predominantly observed null associations ${ }^{(14,15)}$. In contrast, in a Portuguese study, Durão et al. ${ }^{(14)}$ found that an energy-dense foods dietary pattern was associated with higher BMI and higher WHtR in girls but not boys. It is difficult to know whether this differing finding represents the truth (in that their thirty-fiveitem FFQ was more detailed than ours) or a chance association (as it is unclear why girls but not boys would experience the effects of poor diet).

\section{Meaning of the study for clinicians and policymakers}

Our results indicate that dietary scores and patterns derived from brief measures do not substantially drive body composition. This implies that short dietary measures tapping into quality rather than quantity may not help to manage or understand the childhood obesity epidemic. However, they could have other immediate or longer-term benefits for health. The dilemma is that such measures may be close to the limit of what can feasibly be asked clinically and in multifocused population studies that also need precise measurement of a wide range of other exposures and outcomes. Specifically, it is likely that the quantities, rather than frequencies, of consuming different types of food are important for predicting body composition. Similarly, BMI $z$-score and WHtR might be more predictive of quantity, rather than frequency, of dietary intake.

\section{Unanswered questions and future research}

Despite the strengths of our short dietary measure, our study would need to be replicated with more comprehensive and accurate measures before forming recommendations. While these might include existing computerised $24 \mathrm{~h}$ dietary recalls or mobile phone apps ${ }^{(45,46)}$, it remains the case that clinical and public health tools are woefully inadequate to capture energy intake accurately. Developing accurate dietary tools that are feasible for clinical and population use must be amongst the highest priority for obesity research. There is a critical need for research and development if we are to make progress in this area. Considerations include the need to capture habitual, rather than $24 \mathrm{~h}$, diet, and to comprehensively measure food quantity, portion size and absolute energetic intake; all of these are currently out of reach for large studies. Moreover, it would also be valuable to assess associations of dietary trajectories with other health outcomes, including metabolic and inflammatory profiles, 
and whether these associations differ according to weight status (e.g. obese $v$. overweight $v$. underweight).

\section{Conclusion}

In conclusion, dietary scores and patterns derived from brief measures do not appear to substantially drive body composition; nor does BMI $z$-score or WHtR appear to influence dietary quality in subsequent waves at any age. This implies that short dietary measures tapping into quality rather than quantity may not help to manage or understand the childhood obesity epidemic. We believe that developing much more accurate dietary tools for children must be an absolute priority to advance research and practice in childhood obesity.

\section{Acknowledgements}

This paper uses unit record data from Growing Up in Australia, the LSAC. The study is conducted in partnership between the Department of Social Services (DSS), the Australian Institute of Family Studies (AIFS) and the Australian Bureau of Statistics (ABS). The findings and views reported in this paper are those of the authors and should not be attributed to DSS, AIFS or the ABS. The authors thank all the parents and children who took part in the LSAC. The authors would also like to thank Dr Elasma Milanzi (University of Melbourne) for her advice on structural-equation modelling used in this paper.

The authors of this work were supported by the Australian National Health and Medical Research Council (F. K. M., Career Development Fellowship 1111160), (R. C., Postgraduate Scholarship 1133054), (M. W., Senior Research Fellowship 1046518); an Australian Government Research Training Program Scholarship (C. E. G.); and a Murdoch Children's Research Institute PhD Top Up Scholarship (C. E. G.). Research at the Murdoch Children's Research Institute is supported by the Victorian Government's Operational Infrastructure Support Program. The Australian National Health and Medical Research Council, Australian Government, Murdoch Children's Research Institute and the Victorian Government had no role in the design, analysis or writing of this article. The LSAC is funded by the Commonwealth Government of Australia.

The author's contributions are as follows: C. E. G., F. K. M., S. A. C. and M. W. formulated the research questions; C. E. G. designed the research, with input from F. K. M., S. A. C., J. A. K., R. C. and M. W.; C. E. G. conducted the research; C. E. G. analysed the data, with advice and supervision from F. K. M., S. A. C., J. A. K., R. C. and M. W.; and C. E. G. wrote the article, with input from F. K. M., S. A. C., J. A. K., R. C. and M. W. All authors read and approved the final version of the manuscript.

M. W. receives hourly financial reimbursement from the Australian Government for her advisory role on the LSAC. C. E. G., F. K. M., S. A. C., J. A. K. and R. C. declare no potential conflicts of interest.

\section{Supplementary material}

For supplementary material/s referred to in this article, please visit https://doi.org/10.1017/S0007114518003173

\section{References}

1. Ng M, Fleming T, Robinson M, et al. (2014) Global, regional, and national prevalence of overweight and obesity in children and adults during 1980-2013: a systematic analysis for the Global Burden of Disease Study 2013. Lancet 384, 766-781.

2. Johnson BJ, Bell LK, Zarnowiecki D, et al. (2017) Contribution of discretionary foods and drinks to Australian children's intake of energy, saturated fat, added sugars and salt. Children (Basel) 4, E104.

3. Sim LA, Lebow J, Wang Z, et al. (2016) Brief primary care obesity interventions: a meta-analysis. Pediatrics $\mathbf{1 3 8}$, e20160149.

4. Wake M (2018) The failure of anti-obesity programmes in schools. BMJ 360, k507.

5. Jacquier M, Soula HA \& Crauste F (2015) A mathematical model of leptin resistance. Math Biosci 267, 10-23.

6. Friedman J (2014) 20 years of leptin: leptin at 20: an overview. I Endocrinol 223, T1-T8.

7. Santos S, Severo M, Lopes C, et al. (2018) Anthropometric indices based on waist circumference as measures of adiposity in children. Obesity (Silver Spring) 26, 810-813.

8. Malik VS, Pan A, Willett WC, et al. (2013) Sugar-sweetened beverages and weight gain in children and adults: a systematic review and meta-analysis. Am J Clin Nutr 98, 1084-1102.

9. Gasser CE, Mensah FK, Russell M, et al. (2016) Confectionery consumption and overweight, obesity, and related outcomes in children and adolescents: a systematic review and metaanalysis. Am J Clin Nutr 103, 1344-1356.

10. Michels KB \& Schulze MB (2005) Can dietary patterns help us detect diet-disease associations? Nutr Res Rev 18, 241-248.

11. Okubo H, Crozier SR, Harvey NC, et al. (2015) Diet quality across early childhood and adiposity at 6 years: the Southampton Women's Survey. Int J Obes (Lond) 39, 1456-1462.

12. Meyerkort CE, Oddy WH, O'Sullivan TA, et al. (2012) Early diet quality in a longitudinal study of Australian children: associations with nutrition and body mass index later in childhood and adolescence. J Dev Orig Health Dis 3, 21-31.

13. Appannah G, Pot GK, Huang RC, et al. (2015) Identification of a dietary pattern associated with greater cardiometabolic risk in adolescence. Nutr Metab Cardiovasc Dis 25, 643-650.

14. Durão C, Severo M, Oliveira A, et al. (2017) Association between dietary patterns and adiposity from 4 to 7 years of age. Public Health Nutr 20, 1973-1982.

15. Durão C, Severo M, Oliveira A, et al. (2015) Evaluating the effect of energy-dense foods consumption on preschool children's body mass index: a prospective analysis from 2 to 4 years of age. Eur J Nutr 54, 835-843.

16. National Health and Medical Research Council (2013) Australian Dietary Guidelines. Canberra: National Health and Medical Research Council.

17. Norton A \& Monahan K (2015) Wave 6 Weighting and Nonresponse, LSAC Technical Paper no. 15. Greenway: Australian Government Department of Social Services.

18. Australian Institute of Family Studies (2016) Frequently asked questions - Growing Up in Australia: the Longitudinal Study of Australian Children (LSAC). http://www.growingupinaustralia. gov.au/about/faq.html (accessed December 2017).

19. Soloff C, Lawrence D \& Johnstone R (2005) Sample Design, LSAC Technical Paper no. 1. Melbourne: Australian Institute of Family Studies.

20. Australian Institute of Family Studies (2018) Ethics committee and role I Australian Institute of Family Studies. https://aifs. gov.au/ethics-committee-and-role (accessed June 2018). 
21. Australian Institute of Family Studies (2018) Data collection modes by wave I Growing Up in Australia. https://growingu pinaustralia.gov.au/data-and-documentation/data-collectionmodes-wave (accessed August 2018).

22. Australian Institute of Family Studies (2015) Longitudinal Study of Australian Children Data User Guide - November 2015. Melbourne: Australian Institute of Family Studies.

23. Gasser CE, Kerr JA, Mensah FK, et al. (2017) Stability and change in dietary scores and patterns across six waves of the Longitudinal Study of Australian Children. Br J Nutr 117, $1137-1150$.

24. University of California, Los Angeles (UCLA) Institute for Digital Research and Education (2017) How can I perform a factor analysis with categorical (or categorical and continuous) variables? I Stata FAQ - IDRE Stats. https://stats.idre. ucla.edu/stata/faq/how-can-i-perform-a-factor-analysis-withcategorical-or-categorical-and-continuous-variables/ (accessed December 2017)

25. Costello AB \& Osborne JW (2005) Best practices in exploratory factor analysis: four recommendations for getting the most from your analysis. Pract Assess Res Eval 10, 1-9. http:// pareonline.net/pdf/v10n7.pdf (accessed December 2017).

26. Kuczmarski RJ, Ogden CL, Guo SS, et al. (2002) 2000 CDC growth charts for the United States: methods and development. Vital Health Stat 11, number 246, 1-190.

27. Blakemore T, Strazdins L \& Gibbings J (2009) Measuring family socioeconomic position. Aust Soc Pol 8, 121-168.

28. Renzaho AM, Oldroyd J, Burns C, et al. (2009) Over and undernutrition in the children of Australian immigrants: assessing the influence of birthplace of primary carer and English language use at home on the nutritional status of 4-5year-olds. Int J Pediatr Obes 4, 73-80.

29. Carskadon MA \& Acebo C (1993) A self-administered rating scale for pubertal development. J Adolesc Health 14, 190-195.

30. WHO Multicentre Growth Reference Study Group (2006) WHO Child Growth Standards: Length/Height-for-age, Weight-for-age, Weight-for-length, Weight-for-height and Body Mass Index-for-age: Methods and Development. Geneva: World Health Organization.

31. Australian Bureau of Statistics (2008) Information Paper: An Introduction to Socio-economic Indexes for Areas (SEIFA), 2006, ABS Catalogue no. 2039.0. Belconnen: Australian Bureau of Statistics.

32. Gasser CE, Mensah FK, Kerr JA, et al. (2017) Early life socioeconomic determinants of dietary score and pattern trajectories across six waves of the Longitudinal Study of Australian Children. J Epidemiol Community Health 71, 1152-1160.
33. Johnson L, Mander AP, Jones LR, et al. (2008) Energy-dense, low-fiber, high-fat dietary pattern is associated with increased fatness in childhood. Am J Clin Nutr 87, 846-854.

34. Cutler GJ, Flood A, Hannan PJ, et al. (2012) Association between major patterns of dietary intake and weight status in adolescents. Br J Nutr 108, 349-356.

35. Alsharairi NA \& Somerset SM (2016) Skipping breakfast in early childhood and its associations with maternal and child BMI: a study of 2-5-year-old Australian children. Eur J Clin Nutr 70, 450-455.

36. Perry CP, Keane E, Layte R, et al. (2015) The use of a dietary quality score as a predictor of childhood overweight and obesity. BMC Public Health 15, 581.

37. Ritchie LD, Spector P, Stevens MJ, et al. (2007) Dietary patterns in adolescence are related to adiposity in young adulthood in black and white females. J Nutr 137, 399-406.

38. Golley RK, Hendrie GA \& McNaughton SA (2011) Scores on the dietary guideline index for children and adolescents are associated with nutrient intake and socio-economic position but not adiposity. J Nutr 141, 1340-1347.

39. Golley RK, Smithers LG, Mittinty MN, et al. (2013) Diet quality of U.K. infants is associated with dietary, adiposity, cardiovascular, and cognitive outcomes measured at 7-8 years of age. J Nutr 143, 1611-1617.

40. Sanson A, Nicholson J, Ungerer J, et al. (2002) Introducing the Longitudinal Study of Australian Children (LSAC), Discussion Paper no. 1. Melbourne: Australian Institute of Family Studies.

41. Dwyer JT \& Coleman KA (1997) Insights into dietary recall from a longitudinal study: accuracy over four decades. $A m J$ Clin Nutr 65, Suppl. 4, S1153-S1158.

42. Garaulet M, Martínez A, Victoria F, et al. (2000) Difference in dietary intake and activity level between normal-weight and overweight or obese adolescents. J Pediatr Gastroenterol Nutr 30, 253-258.

43. Gasser CE, Mensah FK, Clifford SA, et al. (2018) Parental health behaviour predictors of childhood and adolescent dietary trajectories. Public Health Nutr 21, 1874-1885.

44. Yoo EG (2016) Waist-to-height ratio as a screening tool for obesity and cardiometabolic risk. Korean J Pediatr 59, 425-431.

45. Foster E, Hawkins A, Delve J, et al. (2014) Reducing the cost of dietary assessment: self-completed recall and analysis of nutrition for use with children (SCRAN24). J Hum Nutr Diet 27, Suppl. 1, 26-35.

46. Bejar LM, Sharp BN \& García-Perea MD (2016) The e-EPIDEMIOLOGY mobile phone app for dietary intake assessment: comparison with a food frequency questionnaire. JMIR Res Protoc 5, e208. 